\title{
Horizons/Théâtre
}

Revue d'études théâtrales

\section{Écrits pour la parole de Léonara Miano, mis en scène par Eva Doumbia : un spectacle communautaire ou afropéen?}

Majorie Bertin

\section{(2) OpenEdition}

1 Journals

Édition électronique

URL : https://journals.openedition.org/ht/1705

DOI : $10.4000 /$ ht. 1705

ISSN : 2678-5420

Éditeur

Presses universitaires de Bordeaux

Édition imprimée

Date de publication : 1 janvier 2019

Pagination : 126-132

ISBN : 979-10-300-0718-3

ISSN : 2261-4591

Référence électronique

Majorie Bertin, «Écrits pour la parole de Léonara Miano, mis en scène par Eva Doumbia : un spectacle communautaire ou afropéen? », Horizons/Théâtre [En ligne], 14 | 2019, mis en ligne le 01 juin 2022, consulté le 30 juin 2022. URL : http://journals.openedition.org/ht/1705 ; DOI : https://doi.org/10.4000/ ht. 1705

Licence Creative Commons

La revue Horizons/Théâtre est mise à disposition selon les termes de la Licence Creative Commons Attribution - Pas d'Utilisation Commerciale - Pas de Modification 4.0 International. 


\section{MAJORIE BERTIN}

Marjorie Bertin est docteure en Études théâtrales, enseignante et chercheuse à l'Université Sorbonne Nouvelle - Paris 3. Membre du Séféa (Scènes Francophones et Écritures de l'Altérité) et de l'IRET (Institut de Recherche en Études Théâtrales) de la Sorbonne Nouvelle, elle travaille sur le théâtre et la chanson dans les pays arabes, la circulation des arts entre le monde arabe et l'Occident, le métathéâtre et, plus généralement, les écritures théâtrales contemporaines des xx et $x x l^{e}$ siècles. Elle enseigne au sein de l'Institut d'Études Théâtrales et du Département d'italien de la Sorbonne Nouvelle. Elle est journaliste aux Magazines de RFI, où elle réalise notamment des chroniques, des portraits et des reportages pour les émissions De vive(s) voix, Vous m'en direz des nouvelles, Autour de la question et RFI Musique. Dans la presse écrite, elle est journalistecritique pour Transfuge, E-tcetera, Alternatives théâtrales et Africultures.

Mail: marjorie.bertin@hotmail.fr

Résumé: Cet article propose d'analyser en quoi devenue pièce de théâtre, Écrits pour la parole de l'autrice Léonora Miano peut être perçue comme une tentative " $d^{\prime}$ habiter la frontière » par deux Afropéennes lla Camerounaise d'expression française Léonora Miano et le metteuse en scène française d'origine malienne Eva Doumbial qui questionnent la notion d'identité. Ce spectacle s'inscrirait alors dans la même mouvance que bon nombre d'écritures dramatiques africaines contemporaines qui partagent ce que Sylvie Chalaye analyse, dans Afrique noire et dramaturgies contemporaines : le syndrome Frankenstein', comme un refus d'être identifié pour des artistes qui marronnent et «se jouent de tous les

ABstract: This article focuses ont the work of two leading figures in theater and culture in France: Léonora Miano and Eva Doumbia. It takes the play Écrits pour la parole from Léonora Miano, as case studies, in order to describe and analyse how it's can be taken as an attempt to "habiter la frontière" by two Afropean women (French and Cameroonian Léonora Miano and French director, malian's origin Eva Doumbia) who question the notion masques qui leur sont offerts et qui puisent dans un patrimoine culturel mondial ${ }^{2}$ » sans tomber dans l'universalisme.

Plus largement, cet article esquisse une définition de l'afropéïsme, un concept récent en France, déjà bien aux États-Unis et dans les pays francophones.

Mots-clés: afropéisme, universalisme, frontière, colonisation, féminisme, identité, communautarisme.

1. Sylvie Chalaye, Afrique noire et dramaturgies contemporaines : le syndrome Frankenstein, Éditions théâtrales, coll. «Passages francophones », Paris, 2004.

2. Ibid., p. 108-109.

of identity. Moreover, this article outlines a definition of Afropeism, a recent concept in France, already well established in the United States and in French-speaking countries.

KEYWORDS : afropeism, universalism, border, colonization, identity, feminism, communitarianism. 


\section{Écrits pour la parole de Léonara Miano, mis en scène par Eva Doumbia : un spectacle communautaire ou afropéen?}

Chaque génération doit dans une relative opacité découvrir sa mission, la remplir ou la trahir... Il nous faut perdre l'habitude, maintenant que nous sommes au cour du combat, de minimiser l'action de nos pères ou de feindre l'incompréhension devant leur silence et leur passivité.

Avec ÉCRITS POUR LA PAROle QU'Elle PRÉSEntait dans une nouvelle version au Festival d'Avignon 2017, la metteure en scène franco-ivoiro-malienne Eva Doumbia proposait une pièce sur la visibilité des femmes noires en France aujourd'hui. Constituée à partir de fragments de l'auteure camerounaise d'expression française Léonora Miano, Écrits pour la parole met en scène des Européennes d'origine subsharienne, des « Afropéennes ».

L'action de ce spectacle se situe dans un restaurant, dispositif idoine pour la circulation de la parole théâtrale, où se retrouvent des amies. Ces femmes, dont on ne connait pas l'origine précise, bien que l'on devine qu'elles sont afrodescendantes, y portent des paroles sur la condition de la « femme noire ». Une expression qui revient souvent au sein de la pièce et n'est pas sans rappeler le célèbre poème éponyme de Léopold Sedar Senghor ${ }^{1}$ dans lequel l'identité de la «femme noire » se construit de manière interrogative, perçue à travers l'expression du désir et de l'amour maternel d'un homme. Les deux précédentes versions de ce spectacle, qui s'appelaient alors Afropéennes, ont pourtant parfois été taxées de communautaristes et de racistes ${ }^{2}$ et, de manière générale, la metteure en scène peine à faire programmer ce spectacle. Pourtant, comme s'en défend Eva Doumbia, cette pièce voulait, et continue de vouloir, s'adresser à toutes les femmes, qu'elles soient noires ou non ${ }^{3}$. Par ailleurs, Léonora Miano est également l'auteure D'habiter la frontière dans lequel elle invite Européennes et Afrodescendantes à regarder et à assumer leur histoire commune, sillon dans lequel Eva Doumbia dit également s'inscrire. 
Cet article propose d'analyser en quoi, porté pour la scène et devenu pièce de théâtre, Écrits pour la parole peut être également perçu comme une tentative « d'habiter la frontière » par deux Afropéennes qui questionnent l'identité. Ce spectacle s'inscrirait alors dans la même mouvance que bon nombre d'écritures dramatiques africaines contemporaines qui partagent ce que Sylvie Chalaye analyse, dans Afrique noire et dramaturgies contemporaines : le syndrome Frankenstein4 comme un refus d'être identifié pour des artistes qui marronnent et « se jouent de tous les masques qui leur sont offerts et qui puisent dans un patrimoine culturel mondial ${ }^{5} \gg$ sans tomber dans l'universalisme.

Plus largement, l'objectif de cet article est de présenter ce concept récent, moins aux États-Unis qu'il ne l'est en France et dans les pays francophones, qu'est l'afropéïsme.

Mais revenons à Léonora Miano. Cette autrice camerouno-française d'expression française est née en 1973, à Douala. Installée depuis 1991 en France, elle fait partie des auteurs africains ou/et afros-descendants dont l'écriture désinhibée est traversée par des problématiques liées au racisme, au communautarisme et aux notions de frontières. Bien que très primée en France, Léonora Miano est loin d'être une auteure consensuelle qui revendique aussi bien le droit d'aborder les problèmes de la France que ceux de l'Afrique subsaharienne sans pour autant, comme elle le précise, qu'il faille nécessairement $y$ voir un réquisitoire intransigeant contre le continent :

[...] il est arrivé qu'on considère ma littérature, lorsqu'elle présentait un décor subsaharien, comme une sorte de propos accusatoire à l'égard de l'Afrique subsaharienne et de ses populations. Il n'en est rien, évidemment. Ce qu'il aurait fallu comprendre, c'est mon appartenance à une génération de Subshariens suffsamment bien dans leur peau et assez matures pour se sentir capables d'explorer les zones les plus ténébreuses de leur expérience ${ }^{6}$.

La dernière version spectacle d'Eva Doumbia s'appuie sur plusieurs textes de Léonora Miano, Écrits pour la parole ${ }^{7}$ bien sûr, mais aussi Blues pour Élise 8 . Dans ce roman, qui avait pour vocation de « montrer la France comme vous ne l'avez jamais vue », selon les dires de son auteure, les personnages principaux sont des amies afro-descendantes, des Françaises qui se sont donné le surnom de «Bigger than life », et que le narrateur décrit comme :

[...] intelligentes, financièrement autonomes, belles, chacune à sa manière. Elles s'étaient donné ce nom il y avait déjà des années, quand elles n'étaient encore que des étudiantes souvent désargentées, filles de personne d'important, portant des 
prénoms non alignés, des patronymes à l'ancrage lointain. Bigger than life était devenu leur devise. Elles ne seraient pas toujours plus fortes que l'adversité, mais elles seraient tenaces.

Ces Afropéennes ne sont pas nées dans un même pays, mais simplement au sein d'un même continent; elles ont des points communs culturels bien sûr, mais sont également françaises. Or, Léonora Miano, qui revendique sa propre hybridité culturelle dans ses essais et ses conférences, l'affirme : l'identité « africaine » n'existe pas, pas plus d'ailleurs que le « théâtre » africain. Et pour le coup, la pensée de ceux qui voudraient faire exister à tout prix un africanisme ne serait peut-être pas exempte d'une simplification réductrice que Sylvie Chalaye qualifie pour sa part de «taxidermiste » :

[...] Pourquoi se cramponner à une spécificité africaine qui ferait l'esthétique particulière d'un théâtre? Il faut croire que le regard occidental reste attaché à une volonté taxidermiste de conserver cette image de l'Afrique qui a nourri ses rêves d'enfance, Afrique des féticheurs, des sorciers, Afrique des masques et des petits villages... L'idée d'un théâtre africain nous renvoie immédiatement à ces visions exotiques avec case, raphia, plumes, tam-tams, baobas, souvenirs des vignettes que les petits écoliers traquaient au fond des tablettes de chocolat pour les coller dans un magnifique album Connaissance du monde .

En effet, comme ne cesse également de l'affirmer le philosophe camerounais Achille Mbembe, «l'identité africaine n'existe pas en tant que substance. La nature de cette culture se constitue, dans des formes variées, à travers une série de pratiques ${ }^{10} \gg$.

On retrouve donc ce type de personnages dans Écrits pour la parole dont les protagonistes sont bien issus de ce tissage, de ce métissage. Ce sont des Afropéennes. Selon Pénélope Dechaufour, le terme apparaît pour la première fois sous la plume de l'écrivain kényan Ngugi wa Thiong'o qui explique, dans Décoloniser l'esprit, que le premier marqueur de l'afropéanisme se situe au niveau linguistique.

Ce que nous avons créé, dit-elle, est une tradition hybride, fragile, minoritaire, qu'on peut au mieux qualifier d'afro-européenne, la littérature écrite par des Africains d'expression européenne. Elle a donné de nombreux écrivains et de grandes œuvres : Chinua Achebe, Wole Soyinka, Ayi Kwei Armah, Sembène Ousmane, Léopold Sedar Senghor et beaucoup d'autres ${ }^{11}$.

Cet adjectif qualifie le fait d'être noir et né en Europe ou d'y avoir grandi. Il pose une question cruciale, qui fait encore fréquemment polémique aujourd'hui : comment dire la présence noire sur le continent européen ? Selon Léonora Miano, elle-même, qui a popularisé le terme en France : 
Le terme « afropéen » cherche à décrire ces personnes d'ascendance subsaharienne ou caribéenne et de culture européenne: des individus qui mangent certes des plantains frits mais dont les particularismes ne sont pas tellement différents de ceux qu'on peut trouver dans les régions de France ${ }^{12}$.

Ce terme naît donc d'une contrainte, en France, mais peut-être également en Europe, où nous ne sommes hélas pas toujours loin, comme l'affirment certains Afropéens, de l'époque où un metteur en scène demandait à Jean Genet d'écrire pour les Noirs. La metteure en scène de ces Écrits pour la parole, Eva Doumbia, est elle aussi une Afropéenne : franco-ivoiro-malienne, elle travaille des formes scéniques hybrides, mêlant volontiers le cabaret, le théâtre et le chant. Les personnages de son spectacle, constitué à partir d'un montage de textes de Léonora Miano donc, ne sont pas soumises. Ni victimes, ni militantes, elles s'interrogent pourtant sur le regard que l'on porte sur elles, depuis leur enfance, en raison de leur couleur. Ainsi le personnage de Jézabel raconte-t-elle ce que lui disait sa mère lorsqu'elle était petite fille et rêvait d'être blanche. «N'oublie pas ta couleur, tiens-toi bien. » Avec quelques variantes : «n'oublie pas ta couleur, surveille ton langage, travaille davantage, ne crois pas aux mirages ${ }^{13} \gg$. Puisqu' il est impossible d'oublier sa couleur. Un constat que fera un autre personnage ensuite, pour expliquer pourquoi, selon elle, la « femme noire de France » a été obligée de se construire une image exaltant les représentations sociales - souvent racistes - de l'époque :

Il faut bien voir que la femme noire de France n'a eu pour se rêver s'élever que les bananes les grimaces les roulements d'yeux de Joséphine la nudité mise en cage de Grace qui n'étaient pas françaises Les Françaises noires n'étaient pas assez bien Je ne veux pas choquer en précisant qu'il leur a fallu jouer les sauvages Il leur a fallu jouer les sauvages pour arriver en haut de l'affiche Il leur a fallu jouer les sauvages émoustiller le désir malsain qu'on avait de la sauvage pour arriver en haut de l'affiche Il leur a fallu jouer les sauvages émoustiller le désir malsain qu'on avait de la sauvage habiter une Afrique fabriquée par la pensée raciste pour arriver en haut de l'affiche et gagner leurs galons d'icônes On n'a pas pu s'identifier à elles qui étaient autres construites comme autres ${ }^{14}$.

Ces propos de Léonora Miano ne sont pas, bien sûr, sans échos lointains avec Les Nègres de Jean Genet, dans lesquels ce n'est justement aussi que par le travestissement et le grossissement que les personnages parviennent à s'extraire de leur image. En effet, une partie des Noirs de la pièce y est masquée de blanc, mais l'autre partie se travestit peu à peu à son tour, s'enduisant le visage de cirages de nuances différentes comme pour égarer le spectateur, 
tout en lui faisant entendre que « les Nègres », pour reprendre le titre de la pièce, ne sont pas un groupe distinct et identifiable comme un troupeau de moutons, mais une multitude d'histoires et d'identités qui se meuvent et pensent elles aussi. Par ces procédés, et par le scandale que suscita la première mise en scène de la pièce par Roger Blin, Jean Genet est bel et bien parvenu à inquiéter son spectateur blanc, suffisamment pour que ce dernier soit amené à s'interroger sur son rapport à l'altérité, et particulièrement aux Noirs de la vie réelle.

Or sur scène, dans Écrits sur la parole, c'est bien le contraire que nous montre Eva Doumbia qui nous invite à regarder cette différence des corps de la diaspora sans jamais les caricaturer.

Elle donne à voir des comédiennes issues de la diversité, dont les propos soulignent que leurs problématiques ici ne sont finalement guère différentes des Françaises blanches. La metteure en scène montre des corps loin des clichés qui ne sont pas forcément ordonnés, qui ne révèlent pas toujours ce que l'on attend d'eux, des personnalités et des origines qui ne sont pas semblables. Cette démarche, à la fois esthétique et littéraire - puisqu'il s'agit d'un montage et collage de textes de Miano appartenant à des genres différents - correspond bien, comme le souligne Sylvie Chalaye, à la signature esthétique globale d'Eva Doumbia :

L’esthétique du plateau chez Eva Doumbia est une esthétique du désordre de l'éclatement, une dispersion qui dit l'attachement diasporique des corps. Ces femmes noires sur un plateau qui changent sur leur vécu, leurs expériences, ce n'est pas du communautarisme, mais le meilleur moyen d'amener les femmes européennes. Tenter de comprendre l'autre, un autre vécu de femme, enlever la robe-carcan de la femme noire pour voir LA femme, une femme, à laquelle il ne s'agit pas de s'identifier mais qu'il convient simplement d'envisager au sens fort du terme et non plus de fantasmer ${ }^{15}$.

La différence en terme de représentations socioculturelles apparaît donc comme un «plus ». Les problématiques des personnages féminins que sont le travail, les hommes, la politique et leur famille pour l'essentiel, ne sont pas différentes de celles d'autres européennes. Simplement, leur bagage culturel est autre ou « mixé » et loin de générer un creux, le fait d'《 habiter la frontière $\gg$, pour reprendre le titre de l'essai de Léonora Miano, est un enrichissement des compositions surprenantes qui constituent le divers, tel qu'Édouard Glissant le définit, à savoir « les différences qui se rencontrent, s'ajustent, s'opposent, s'accordent et produisent de l'imprévisible ${ }^{16} \gg$. 


\section{Notes}

1. Léopold Sédar Senghor, «Femme noire », Chants d’ombres, Paris, Le Seuil, 1956.

2. Ayann Koudou, "Afropéennes" : théâtre communautaire ? ", Le Point [En ligne]. Mis en ligne le 20/02/2015. Disponible sur : http://www.lepoint.fr/culture/ afropeennes-theatre-communautaire-20-02-2015-1906582_3.php. Consulté le 11/02/2021.

3. Yvan Amar, «Écrits pour la parole de Léonora Miano, mis en scène par Eva Doumbia », RFI [En ligne]. Mis en ligne le 17/07/2017. Disponible sur : http://www.rfi.fr/emission/20170715-avignon-2017-leonora-miano-eva-dumbia. Consulté le 11/02/2021.

4. Sylvie Chalaye, Afrique noire et dramaturgies contemporaines : le syndrome Frankenstein, Éditions théâtrales, coll. «Passages francophones », Paris, 2004.

5. Ibid., p. 108-109.

6. Léonora Miano, Habiter la frontière, Paris, L’Arche, coll. «Tête-à-tête », 2012, p. 5-6.

7. Léonora Miano, Écrits pour la parole, Paris, L'Arche, coll. « Scène ouverte », 2012.

8. Léonora Miano, Blues pour Élise, Plon, Paris, 2010.

9. Sylvie Chalaye, op. cit., p. 21.

10. Achille Mbembe, «À propos des écritures africaines de soi ", Politique africaine $\mathrm{n}^{\circ} 77$, mars 2000, p. 42.

11. Ngugi wa Thiong'o, Décoloniser l'esprit, Paris, La fabrique éditions, 2011, p. 58.

12. Léonora Miano, entretien pour Le Magazine littéraire, 2010.

13. Op. cit.

14. Léonora Miano, Écrits pour la parole, op. cit.

15. Sylvie Chalaye, «Eva Doumbia sur Afropéennes de Léonora Miano. Corps diasporique, corps inouï Festival des Francophonies en Limousin, édition 2012 ", Africultures, n 99-100, Afropéa, un territoire culturel à inventer, 2014, p. 202-205.

16. Édouard Glissant, Introduction à une poétique du divers, Paris, Gallimard, 2006 [1990], p. 98. 